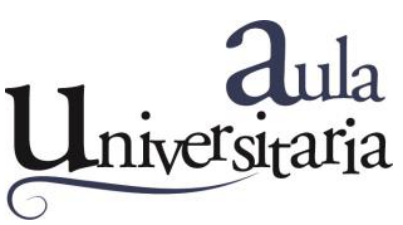

\title{
Licenciatura en administración de la salud: un estudio de sus graduados en el período 2010-2018
}

\author{
BONOMO, Emanuel D.; PAULINI, Rosario B.; DEZAR, Gimena V. ${ }^{3}$
}

\begin{abstract}
Filiaciones institucionales
${ }^{1}$ Alumno de la carrera de Licenciatura en Administración de la Salud. Escuela Superior de Sanidad Dr. Ramón Carrillo. Facultad de Bioquímica y Ciencias Biológicas. Universidad Nacional del Litoral.

${ }^{2}$ Asistente de Coordinación de Relaciones Internacionales. Facultad de Bioquímica y Ciencias Biológicas. Universidad Nacional del Litoral.

${ }^{3}$ Directora de Extensión Social y Cultural. Facultad de Bioquímica y Ciencias Biológicas. Universidad Nacional del Litoral.
\end{abstract}

\section{Correspondencia}

Paraje El Pozo, s/n, 1er. Piso. Santa Fe de la Vera Cruz, Santa Fe, Argentina. Tel. 54-342-4575210

emanuel_bono@hotmail.com

\section{Resumen}

En el año 1992 se crea la carrera de grado de Licenciatura en Administración de Salud de la Escuela Superior de Sanidad (ESS) Dr. Ramón Carrillo dependiente de la Facultad de Bioquímica y Ciencias Biológicas (FBCB) de la Universidad Nacional del Litoral (UNL). La presente investigación tiene como objetivo describir el perfil, lugar de procedencia y la duración de los estudios de los graduados de la Licenciatura en Administración de Salud de la ESS, y la valoración de los mismos sobre la formación de grado recibida. Se realizó una investigación cuanti-cualitativa, de corte transversal y descriptiva. La población de estudio estuvo constituida por 68 profesionales graduados de la carrera Licenciatura en Administración de la Salud de la ESS, FBCB-UNL, en el período 20102018. El instrumento utilizado para obtener datos sobre los graduados fue un cuestionario (formulario de Google Drive), con modalidad para contestar online e individual. Entre los principales resultados del estudio, respecto del perfil de los graduados, se destaca que existe un predominio del género femenino (82,3\%), y provienen del interior de la provincia de Santa Fe. En cuanto a la valoración sobre la formación de grado recibida, la misma fue positiva en su gran mayoría $(87,1 \%)$. Además, un importante graduados en el período 2010-2018. Aula Universitaria $n^{\circ} 21$. e0007, pp. 56-67. DOI: https://doi.org/10.109au.2020.21.e0007 
porcentaje de los graduados continuó formándose luego de finalizar sus estudios de grado $(67,7 \%)$ y se sienten orgullosos de ser graduados de esta casa de estudios.

Se considera de suma importancia continuar con líneas de investigación sobre estudios de seguimiento de egresados para poder contar con datos relevantes y actualizados acerca de la realidad formativa y ocupacional de los mismos.

\section{Palabras clave}

graduados | Licenciatura en Administración de la Salud | valoración de la formación recibida

\section{Summary}

In 1992, the undergraduate degree in the Bachelor's degree for Health Administration in the Superior School of Health (Escuela Superior de Sanidad, ESS) Dr. Ramón Carrillo was created. This school, is a dependancy of the Faculty of Biochemistry and Biological Sciences (FBCB) Universidad Nacional del Litoral (UNL).

The following research aims at describing the profile, place of birth and the years of study of the graduates of the Bachelor's degree in Health Administration of the ESS and their concept, opinion and evaluation regarding their academic formation. A cross-section research was performed with quanti-qualitative and descriptive features. The population under study was made up of 68 graduate professionals of the Career in Health Administration of the ESS, FBCB-UNL, from 2010 to 2018. A questionnaire was given with Google Drive forms, with the possibility for the professionals to do them both, on line or individual completion. Among the most important results of this study (in reference to the graduates'profile) we want to highlight women's predominance (82,3\%), who are mainly coming from the interior of Santa Fe Province. As for the evaluation regarding the academic formation, this turned out to be mainly positive (87.1\%). Besides, an important percentage of graduates kept on receiving academic formation after finishing their graduate studies (67.7\%). All of them feel and show the pride of being part of this University. It is considered highly important to keep on having research lines on follow up studies of our graduates so as to count with relevant and updated data about their academic formation and occupational reality or facts.

\section{Keywords}

graduates | Bachelor's degree for Health Administration | assessment of training received

\section{Introducción}

Los problemas del empleo se han transformado en un tema de debate en todo el mundo. Los cambios tecnológicos y organizacionales de la «sociedad del conocimiento» demandan trabajadores cada vez con más saberes y más competencias (Ardenghi, 2007).

Existen numerosos estudios relacionados con el seguimiento de egresados. Entre los más destacados, es importante mencionar el estudio de investigación que se denominó oficialmente «Educación superior y empleabilidad de los 
titulados en Europa», conocido por sus siglas en inglés como CHEERS. EI estudio se llevó a cabo entre 1998 y 1999 en 37000 graduados universitarios en 1994-1995 de nueve países de la Unión Europea y proporcionó información a través de un cuestionario escrito sobre la relación entre la educación superior cuatro años después de la graduación. Los resultados de este proyecto revelaron una marcada diversidad entre los diferentes países en lo referente a la educación superior y a los vínculos entre esta y el trabajo de graduados universitarios (Mora, 2011).

El proyecto «The Flexible Professional in the Knowledge Society: New Demandson Higher Education in Europe» (REFLEX) se inició en 2006 con la participación de 14 países de Europa y Japón, financiado con recursos del Sexto Programa Marco de la Unión Europea. La actividad principal consistió en la aplicación de una encuesta a graduados universitarios que finalizaron sus estudios en 1999-2000 en los países intervinientes, lográndose una base de datos con un total de 40787 encuestas. El proyecto REFLEX se centró en el análisis de las competencias que requieren los egresados para funcionar adecuadamente en la sociedad del conocimiento; el papel de los centros de educación superior para ayudar a los graduados universitarios a desarrollar las competencias y las tensiones que surgen a medida que graduados universitarios, centros de educación superior, empresarios y otras figuras importantes se esfuerzan en alcanzar sus propios objetivos y formas de resolverlas (Riquelme, 2015).

En Argentina, la Universidad Nacional Tres de Febrero, realizó un estudio sobre el mercado de trabajo para egresados universitarios recientes que incluyó a diez universidades nacionales. Se encuestaron a 9630 graduados de 42 carreras de grado y se indagaron aspectos relacionados a la caracterización de las tareas, el desempeño laboral, las competencias, habilidades y destrezas requeridas o valoradas en los puestos de trabajo, entre otros.

A nivel local, en la provincia de Santa Fe, la Facultad de Ciencias Económicas (FCE) de la UNL llevó a cabo un estudio en 107 egresados donde se analizó la situación laboral, la adecuación entre el título obtenido y la ocupación actual, y la evaluación retrospectiva de los estudios de la FCE (Zandomeni y Chignoli, 2006).

En el ámbito de la FBCB se realizó un estudio de graduados de Licenciatura en Nutrición, analizando el período 2010-2013. Participaron voluntariamente 104 graduados donde se analizó: tipo y características de la inserción laboral; características del proceso de inserción, valoración de la formación de grado recibida, formaciones de posgrado y/o capacitación (Paulini, 2016).

Al momento del presente estudio, no se han encontrado en la bibliografía consultada investigaciones sobre los graduados de la carrera Licenciatura en Administración de Salud de la ESS. Surge así el interés de investigar sobre el perfil y la formación de grado recibida por estos profesionales de la salud.

En particular, se espera que la información que se genere impulse procesos de reflexión al interior de la institución y contribuya a sustentar decisiones académicas que permitan superar en forma continua la calidad de la formación 
de los graduados. Además se espera promover espacios de reflexión y diálogo con los mismos, para poder generar instancias de intercambio en pos de las mejoras de la carrera.

\section{Objetivos}

- Describir el perfil, lugar de procedencia y la duración de los estudios de los graduados de Licenciatura en Administración de Salud (GLAS) de la Escuela Superior de Sanidad.

- Describir la valoración de los graduados respecto de la formación de grado recibida.

\section{Metodología}

Se realizó una investigación cuali-cuantitativa, de corte transversal, descriptiva. La población de estudio estuvo constituida por profesionales graduados del plan de estudios vigente del año 2004, de la carrera Licenciatura en Administración de la Salud de la ESS-FBCB-UNL en el período 2010-2018.

Se trabajó con la totalidad de los graduados que culminaron sus estudios entre los años 2010 y 2018. Según el padrón aportado por el Departamento de Alumnado FBCB-UNL, al momento de inicio de la investigación se contó con un número de 68 graduados en el período de análisis, luego la muestra quedó representada por un total de 62 graduados.

El instrumento que se utilizó consistió en un cuestionario que incluyó preguntas cerradas de opción múltiple, preguntas abiertas o semiabiertas. El mismo recogió información como datos generales, duración de los estudios y valoración de la formación de grado recibida, según sus propias trayectorias académicas y percepciones.

La técnica de recolección de la información se realizó mediante un cuestionario a través de formularios de Google Drive, con modalidad para contestar online e individual. Este instrumento estuvo disponible durante un período de tres meses, desde el 16 de diciembre de 2018 hasta el 16 de marzo de 2019. En primera instancia, se realizó un acercamiento al graduado vía email informando sobre el estudio, invitándolo a participar y el medio utilizado para aplicar el cuestionario. En una segunda instancia se procedió a contactarlos por vía telefónica.

Para el tratamiento estadístico de los datos se utilizó la aplicación de hojas de cálculo Microsoft Excel. Se efectuó un detallado análisis descriptivo de las variables estudiadas, los cuales se resumieron en gráficos y proporciones. 


\section{Resultados}

\section{Perfil, lugar de procedencia y duración de los estudios}

Del total de los GLAS que respondieron la encuesta, el 82,3\% (51) es de género femenino, mientras que solo el 17,7\% (11) restante es de masculinos.

Considerando los grupos etarios, el 75,8 \% de GLAS son jóvenes entre 30 y 39 años de edad. Le siguen en orden decreciente los jóvenes entre 20 y 29 años, que representan un $14,5 \%$. Los graduados entre 40 a 49 años totalizaron el 6,5\%. Por último, el 3,2\% tiene 60 años o más.

Respecto de la variable procedencia, es decir, lugar de donde provienen al momento de iniciar la carrera, un $46,8 \%$ de los GLAS proviene del interior de la provincia de Santa $\mathrm{Fe}$, y en un mismo porcentaje $(46,8 \%)$ es de Santa $\mathrm{Fe}$ capital. Solo el 6,5\% proviene de otras provincias (Río Negro y Entre Ríos). Los graduados provenientes del interior de la provincia de Santa Fe corresponden a localidades como Esperanza, San Justo, Santo Tomé, Villa Ocampo, Avellaneda, Barrancas, Ceres, El Trébol, Malabrigo, Marcelino Escalada, María Juana, Recreo, entre otras.

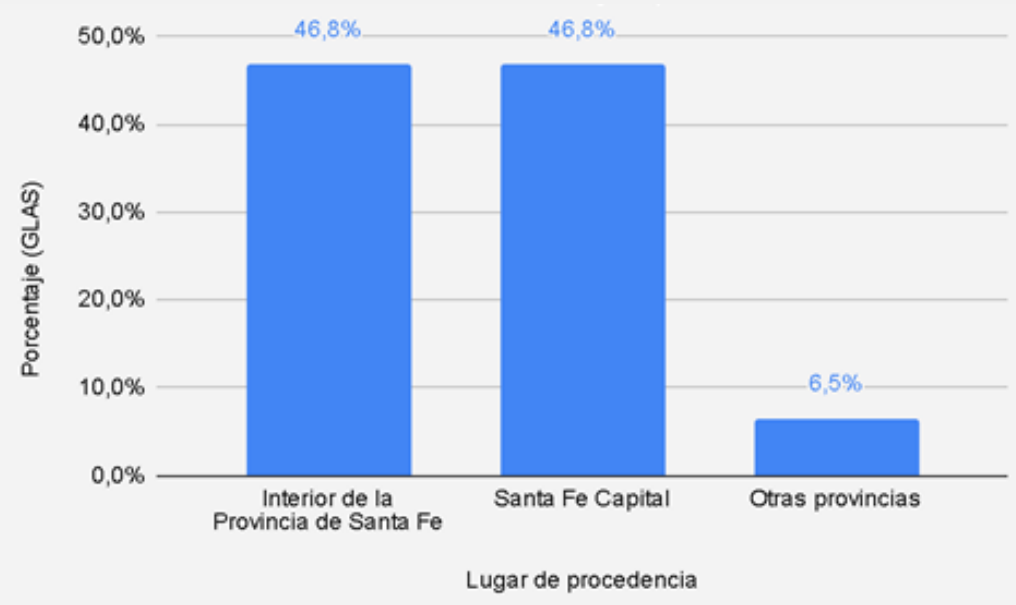

Figura 1. GLAS según procedencia. 2010-2018. ( $n=62)$.

Fuente: elaboración propia a partir de los datos de la encuesta.

En la Figura 2 se observa la distribución de los graduados según la variable año de ingreso. La mayor parte de los encuestados ingresó antes del año 2004 $(38,7 \%)$; le siguen en orden decreciente un $22,6 \%$ que ingresó en $2007 ; 14,5$ $\%$ en 2005 y $12,9 \%$ en 2004 . Tanto en el año 2008 como 2009 se registró un 4,8 \% de ingresantes para cada año. Por último, en 2006 ingresó el 1,6\%. 


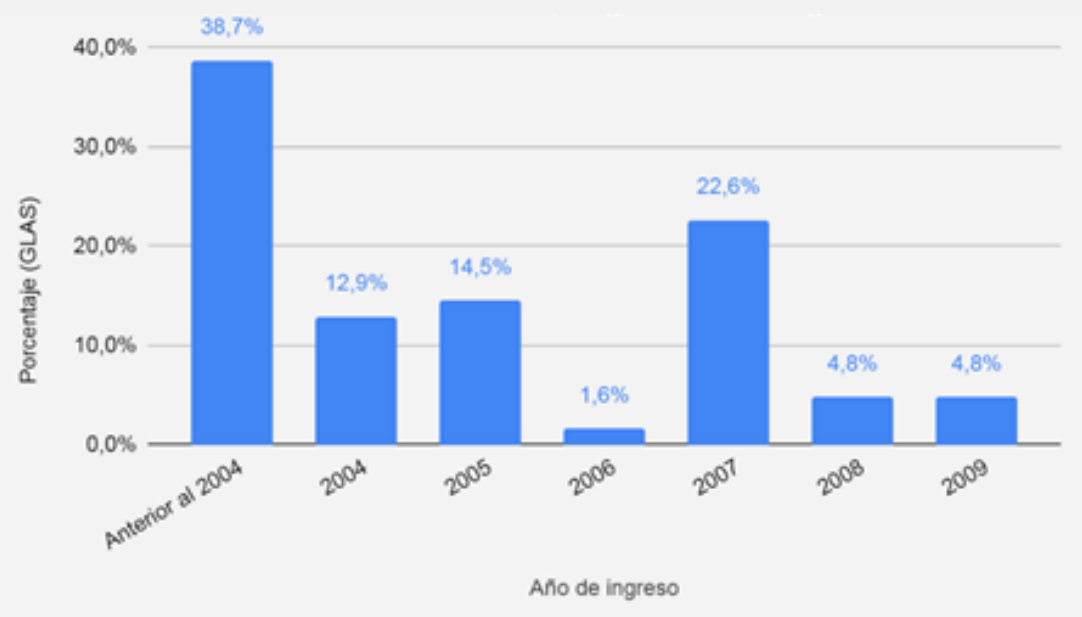

Figura 2. GLAS según año de ingreso. 2010-2018. ( $n=62)$

Fuente: elaboración propia a partir de los datos de la encuesta.

Respecto de la variable año de graduación, en la Figura 3 se muestra que 2013 fue el año de mayor cantidad de graduados, con un $21 \%$; siguen en orden decreciente los años 2012, 2014, 2010-2011, siendo el año 2018 el de menor porcentual.

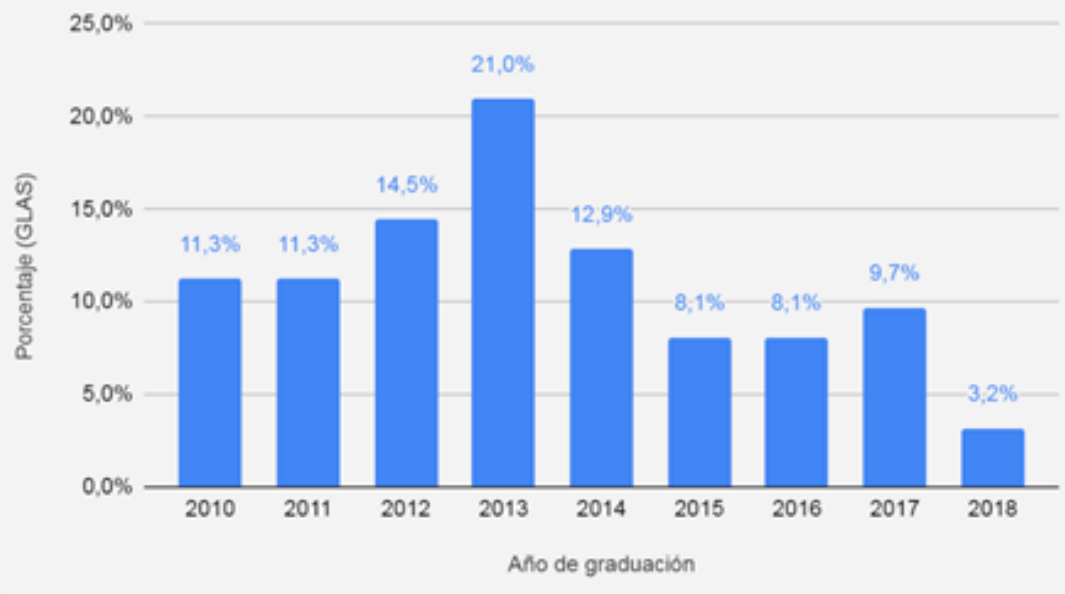

Figura 3. GLAS según año de graduación. 2010-2018. ( $n=62)$

Fuente: elaboración propia a partir de los datos de la encuesta.

En cuanto a la duración de los estudios, el encuestado podía responder de manera voluntaria si realizó sus estudios dentro de lo establecido por el Plan de Estudio de la carrera de Licenciatura en Administración de la Salud de la ESSFBCB-UNL. Si el encuestado no realizó la carrera en el tiempo establecido, se le consultó además en qué momento se produjo el atraso en la misma.

De un total de 62 respuestas, todos los GLAS manifestaron haberse retrasado en la carrera. Un gran porcentaje de los mismos $(75,9 \%)$ dijo haberse retrasado 
en la asignatura Proyecto Final. Cabe destacar que el resto de las respuestas tuvo un porcentaje mucho menor en comparación al año en que se produjo el retraso en la carrera. Solo el 3,2\% de los graduados hizo más lenta toda la carrera.

\section{Descripción de la valoración de la formación de grado recibida}

En este apartado se analiza la valoración de la formación de grado recibida según la trayectoria académica y la percepción de cada uno de los GLAS que respondieron a la encuesta.

Se puede observar en la Figura 4 que la mayoría de los encuestados $(87,1$ $\%)$ la valoró positivamente, en el rango entre muy buena y buena.

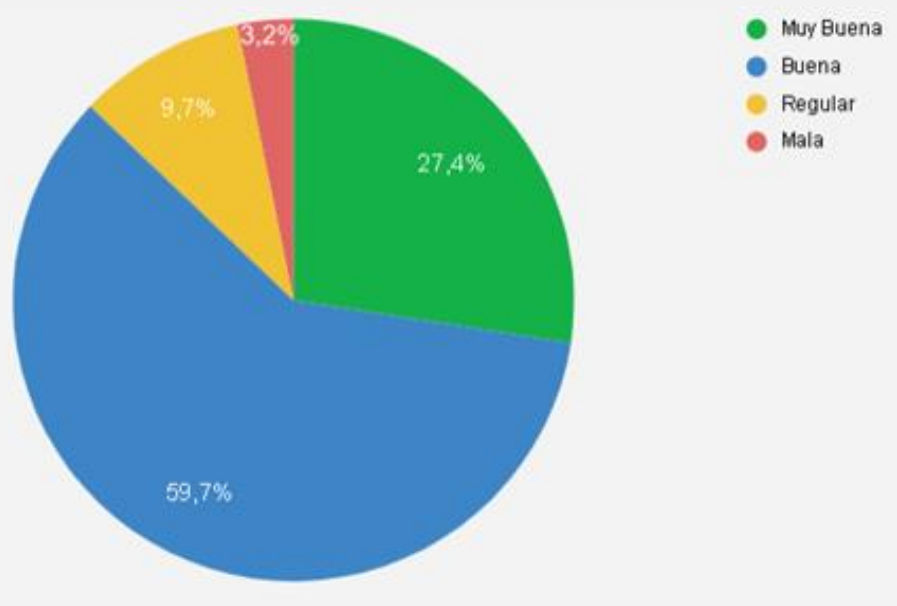

Figura 4. GLAS según valoración de la formación de grado recibida. 2010-2018. ( $n=62)$

Fuente: elaboración propia a partir de los datos de la encuesta.

Al momento de preguntarles: ¿Qué conocimientos o saberes considera que son básicos en el desempeño de su profesión y no se adquirieron o no fueron suficientes durante la carrera? los encuestados respondieron de forma opcional y libremente ya que se trataba de una pregunta abierta, luego se procedió a categorizar las mismas. De un total de 49 respuestas, las más relevantes se mencionan a continuación: un 38,8 \% dijo que facturación es un conocimiento básico en el desempeño de la profesión y no se adquirió o no fue suficiente durante la carrera. Le sigue un $20,4 \%$ que apuntó a las prácticas en terreno y trabajo de campo, y $18,4 \%$ que señaló Contabilidad como materia que no fue suficiente durante la carrera. El 10,2\% de los GLAS hizo hincapié en Auditoría, conocimiento que no fue adquirido durante la carrera. Por último, un $6,1 \%$ de los graduados sostuvo que la asignatura Salud Pública no fue suficiente durante el dictado de la carrera.

Además, los GLAS fueron consultados sobre la percepción de insuficiencias en la formación de grado recibida. Más de la mitad de los encuestados (53,2\%) respondió que hubo insuficiencias en la formación de grado según sus 
percepciones. Un $51,5 \%$ de los graduados dijo que las mismas se debían a programas de estudios inadecuados (planificaciones académicas de las asignaturas), y un $30,3 \%$ a deficiencias que devenían de los docentes (planificaciones y estrategias de enseñanza), y en menor grado de su involucramiento en el estudio. En la Figura 6 se observa el resto de las respuestas de los encuestados conforme a sus percepciones.

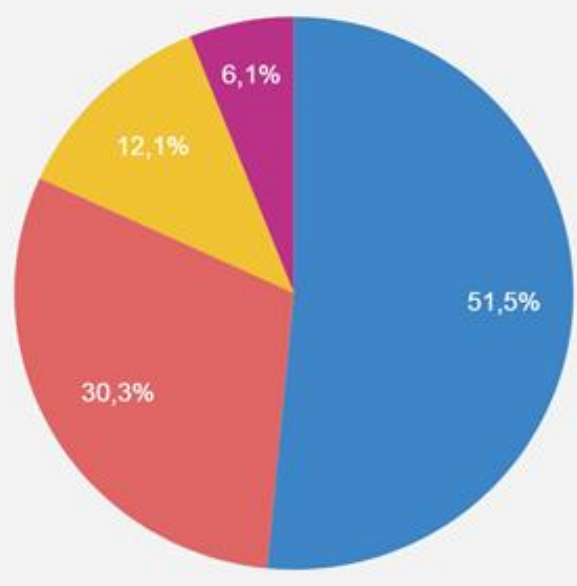

Programa de estudio inadecuado

Deficiencia de los docentes

Dedicación personal

insuficiente al estudio

- Ns/Nc

Figura 6. GLAS según factores atribuidos a las insuficiencias en la formación de grado.

Fuente: elaboración propia a partir de los datos de la encuesta.

También se les consultó si volverían a elegir la misma carrera, y un alto porcentaje $(64,5 \%)$ respondió que sí; un $17,7 \%$, que no; y en la misma proporción no supieron o no contestaron. Cabe destacar que un $80,6 \%$ de los encuestados se sentía orgulloso de ser graduado de la ESS-FBCB-UNL.

Según las percepciones de los GLAS, hay falencias en el plan de estudio de la carrera, falta de contacto entre graduados y falta de apoyo en el Proyecto Final. Además, mencionaron la preocupación por la falta de experiencia al momento de la obtención del título de grado; hicieron referencia a la inexistencia de pautas relacionadas con los plazos de corrección de planes y proyectos finales de la carrera, que afectan directamente al alumno en cuestiones de oportunidades laborales.

Por último, mencionaron la falta de difusión y reconocimiento de la carrera de Licenciatura en Administración de la Salud, como también la necesidad de generar mayores y mejores contenidos para lograr competitividad en el campo de la profesión.

\section{Discusión}

No se han encontrado en la bibliografía consultada trabajos sobre el perfil de los graduados y formación de grado recibida de los graduados de Licenciatura en 
Administración de la Salud. Se comparará este estudio con investigaciones realizadas en graduados de otras carreras universitarias.

Respecto del perfil, lugar de procedencia y duración de los estudios:

Según Zandomeni y Chignoli (2006) en su investigación «Estudio de seguimiento de egresados. El caso de los graduados recientes de la FCE-UNL», el género femenino representa un $65 \%$ de los graduados de dicha unidad académica coincidiendo con el presente estudio donde también existe un predominio del género femenino pero en una mayor proporción (82\%).

En lo que hace al lugar de procedencia, según el informe de 2015 «Panorama de la Universidad en 80 gráficos", los estudiantes de toda la UNL provienen de la provincia de Santa Fe (60\%) y de Entre Ríos un $22 \%$. Sin embargo, los GLAS provienen en una gran mayoría (94\%) del interior de la provincia de Santa Fe y Santa Fe capital.

Con relación a la duración de los estudios, el Plan de la carrera de Licenciatura en Administración de la Salud de la ESS-FBCB-UNL cuenta con una duración de 4 años y seis meses, lo que hace un total de 9 cuatrimestres. En el presente trabajo, todos los encuestados manifestaron haber tenido un retraso en la carrera durante el cursado; en su mayoría (75\%), dijeron haber sufrido dicho atraso en la asignatura Proyecto Final. Esto significaría que la duración de los estudios de todos los GLAS en el período 2010-2018 fue mayor que lo establecido en el Plan de Estudios (Res. Ministerial № 579/2016).

Respecto de la valoración de los graduados sobre la formación de grado recibida, la mayoría de los encuestados (87\%) la valoró positivamente entre muy buena y buena. Resultados similares se hallaron en el estudio antes mencionado de la $\mathrm{FCE}$, donde el $91 \%$ de los encuestados hizo una valoración positiva sobre la formación de grado recibida (Zandomeni y Chignoli, 2006). Por otro lado, en el estudio «La valoración que los graduados tienen sobre su formación universitaria desde su situación laboral actual» solo el $52 \%$ realizó una valoración positiva de la formación universitaria recibida (Sánchez Navarro, 2015).

Para el presente estudio, cuando se consultó a los graduados sobre la percepción de insuficiencias en la formación de grado, más de la mitad de los licenciados en Administración de la Salud de la ESS-FBCB-UNL (53,2 \%) respondió de manera afirmativa y lo atribuyó en su mayoría (51,5\%) a los programas de estudios inadecuados. Esto coincide con lo expresado por Paulini y Dezar (2016), en cuya investigación los graduados de Licenciatura en Nutrición de la FBCB-UNL responden en un 63 \% que las insuficiencias en la formación de grado recibida se deben a los programas de estudios inadecuados.

A la hora de elegir si volverían a estudiar la misma carrera, el $65 \%$ de los licenciados en Administración de la Salud respondió afirmativamente. Nuevamente, esto coincide con lo expresado por los graduados de Licenciatura en Nutrición, donde el $61 \%$ volvería a elegir la misma carrera. Por último, un 81 $\%$ de los graduados de Licenciatura en Nutrición siente orgullo de ser graduados 
de la FBCB-UNL, lo que coincide con los resultados de la presente investigación, donde el $71 \%$ de graduados siente orgullo de ser graduados de la ESS-FBCB-UNL.

\section{Conclusiones}

Con referencia al perfil de los GLAS de la FBCB-UNL, hay un claro predominio de mujeres y en gran proporción tienen entre 30 y 39 años de edad. Esto puede deberse a que las carreras relacionadas con la sanidad recaen más sobre el género femenino producto del restablecimiento de la democracia que permitió mayor apertura de las universidades y el impulso de un proceso de feminización de la medicina y carreras en el área de la salud.

La mayoría de los graduados proviene del interior de la provincia de Santa $\mathrm{Fe}$ y de Santa Fe capital, en igual medida. Solo el $6 \%$ de ellos proviene de otras provincias, lo cual puede deberse a que los alumnos optan por una residencia cercana a la unidad académica donde cursan sus estudios superiores.

En cuanto a la duración de los estudios, gran parte de los GLAS que comprendió el presente trabajo de investigación perteneció a las cohortes del año 2004, pero en su mayoría culminó la carrera en 2013, es decir que una gran proporción de los encuestados no logró realizar la carrera en tiempo establecido por el plan de estudios de la carrera de Licenciatura en Administración de la Salud de la ESS-FBCB-UNL. En su mayoría, mencionan haber tenido un atraso en la carrera en la asignatura Proyecto Final.

En lo referente a la valoración que hacen los graduados de la formación de grado recibida, los mismos realizan sugerencias sobre los conocimientos básicos que necesitarían para el desempeño de su profesión y que sería necesarios adquirir durante la carrera. Los aspectos que mencionan son la incorporación de facturación, prácticas y trabajo de campo. Además, los graduados realizan valoraciones según su trayectoria académica y su percepción, y afirman que existen insuficiencias en la formación de grado recibida atribuida a los programas de estudios inadecuados y las deficiencias de los docentes.

Sin embargo, los graduados, en su mayoría, valoran en forma positiva la formación de grado recibida, volverían a elegir la carrera de Licenciatura en Administración de la Salud ESS-FBCB-UNL y se sienten orgullosos de ser graduados de esta casa de estudios.

\section{Agradecimientos}

A la Universidad Nacional del Litoral, a la Facultad de Bioquímica y Ciencias Biológicas, y a la Escuela Superior de Sanidad, por el apoyo institucional brindado y por permitirnos realizar esta tarea. A los graduados de la carrera de Licenciatura en Administración de la Salud que decidieron colaborar en el presente trabajo. 


\section{Referencias bibliográficas}

- Ardenghi, V. (2007). Trayectorias profesionales y laborales de jóvenes universitarios graduados de la carrera de Artes Plásticas de la Universidad Nacional de La Plata. Departamento de Plástica. Facultad de Bellas Artes, UNLP.

- Ministerio de Educación de la Nación (2016). Proyecto de creación de la carrera de Licenciatura en Administración de la Salud. Universidad Nacional del Litoral. Res. Ministerial $N^{\circ} 579 / 2016$.

- Mora, J. (2011). El profesional flexible en la sociedad del conocimiento: Informe resumen de los resultados del Proyecto PROFLEX en Latinoamérica. CEGES, Centro de Estudios en Gestión de la Educación Superior, Universidad Politécnica de Valencia.

- Paulini, R. (2016). Estudio de graduados de la Licenciatura en Nutrición. Tesis de grado. Universidad Nacional del Litoral, Santa Fe, Argentina.

- Paulini, R.; Dezar, G., (2016). Licenciatura en nutrición de la Universidad Nacional del Litoral: la mirada de sus graduados recientes. Rev. Aula Universitaria, (18), 114-126.

- Riquelme, G.; Magariños, E. (2015). Alternativas de encuestas de egresados universitarios: la experiencia de la UNL. $12^{\circ}$ Congreso Nacional de Estudios del Trabajo.

- Sánchez, A.; Navarro, J. (2016). La valoración que los graduados tienen sobre su formación universitaria desde su situación laboral actual. Rev. Complutense de Educación, 27(2).

- Universidad Nacional del Litoral (2015). Informe de Indicadores 2014. Panorama sobre la Universidad en 80 gráficos. 1ra. ed. ilustrada.

- Zandomeni, N.; Chignoli, S. (2006). Estudio de seguimiento de egresados. El caso de los graduados recientes de la FCE-UNL. Rev. De Cs. Econ., 6(1).

\section{Fuentes electrónicas}

- http://www.seguimientoegresados.com/documents/Analisis_de_las_Competencias_de_los_ Jovenes_Graduados_Universitarios_Espa\%C3\%B1oles.pdf 